\title{
Artroplastia total de codo después de un proceso infeccioso. Cirugía en dos tiempos
}

\author{
Gerardo Gallucci, Javier Sánchez Saba, Jorge G. Boretto, Ezequiel E. Zaidenberg, Agustín Donndorff, \\ Ignacio Rellán, Pablo De Carli \\ Sector de Cirugía de Mano y Miembro Superior, Instituto de Ortopedia y Traumatología “Prof. Dr. Carlos E. Ottolenghi”, \\ Hospital Italiano de Buenos Aires, Ciudad Autónoma de Buenos Aires, Argentina
}

\begin{abstract}
RESUMEN
Objetivo: Comunicar los resultados en pacientes con un proceso infeccioso del codo, tratados en 2 etapas quirúrgicas: la primera con un espaciador de cemento con antibiótico y la segunda con una artroplastia total de codo. Materiales y Métodos: Se describen los criterios de inclusión, el diagnóstico de infección y las 2 etapas quirúrgicas. Resultados: Se incluyeron 10 pacientes (4 hombres y 6 mujeres, edad promedio 62 años). Causas iniciales: degenerativas (2 casos) y traumáticas (8 casos). Se realizaron 4 aloprótesis y 2 colgajos de dorsal ancho. Se identificaron 2 grupos: A (defectos óseos $<4 \mathrm{~cm}$ ) y B ( $>4 \mathrm{~cm}$ ). El seguimiento fue de 5 años. La flexo-extensión fue de $117^{\circ} / 29^{\circ}$ en el preoperatorio y $130^{\circ} / 29^{\circ}$ en el posoperatorio; los puntajes de dolor fueron 6,5 y 2,5 (EAV); 40 y 80 (MEPS); y 56 y 30 (DASH), respectivamente. La fuerza de extensión fue de M5 (4 casos), M4 (2 casos), M3 (1 caso), M1 (2 casos) y M0 (1 caso). Un paciente tuvo una necrosis del colgajo que evolucionó con infección y 9 no tenían infección al final del seguimiento. Los pacientes del grupo A tenían menos cirugías previas y mejores resultados funcionales. Conclusiones: El tratamiento de un proceso infeccioso de codo mediante un espaciador de cemento con antibiótico permite controlar la infección en un alto porcentaje de los casos. La reconstrucción protésica secundaria es demandante y se asocia a complicaciones. Cabe esperar que, cuanto más grande sea el defecto óseo y mayor la cantidad de procedimientos previos, los resultados funcionales sean peores.
\end{abstract}

Palabras clave: Artroplastia de codo; prótesis de codo; infección; osteomielitis; espaciador de cemento.

Nivel de Evidencia: IV

Total Elbow Arthroplasty After an Infectious Process. Two-stage Surgery

\section{ABSTRACT}

Objective: To report the results of patients with an infectious elbow process, treated in 2 surgical stages: the first with an antibiotic cement spacer (ACS) and the second with a total elbow arthroplasty. Materials and Methods: The inclusion criteria, the diagnosis of infection and the 2 surgical stages are described. Results: 10 patients were included ( 7 men and 3 women), average age: 62 years old. Initial causes: degenerative in 2 cases and traumatic in 8.4 alloprostheses and 2 latissimus dorsi flaps were performed. Follow-up was 5 years. Flexo-extension was $117^{\circ} / 29^{\circ}$ in preoperative and $130^{\circ} / 29^{\circ}$ in postoperative; pain according to VAS: 6.5 and 2.5; MEPS: 40 and 80; DASH 56 and 30 respectively. The extension strength was M5 (4 cases), M4 (2), M3 (1), M1 (2) and M0 (1). One patient presented a necrosis of the flap that evolved with infection. In 9 of the 10 cases the patients were free of infection at the end of the follow-up. Two groups of patients were identified: Group A (bone defects less than $4 \mathrm{~cm}$ ) and B (more than $4 \mathrm{~cm}$ ). Group A patients had fewer previous surgeries and better functional outcomes. Conclusion: The treatment of an infectious elbow process through the placement of antibiotic cement spacer, allows a control of the infection in a high percentage of cases. Secondary prosthetic reconstruction is demanding and associated with complications. It is to be expected that the greater the bone defect and the greater the number of previous procedures, the worse the functional results.

Key words: Total elbow arthroplasty; infection; osteomyelitis; antibiotic spacer.

Level of Evidence: IV

Recibido el 19-7-2020. Aceptado luego de la evaluación el 25-8-2020 • Dr. GERARDO GALLUCCI • gerardo.gallucci@ hospitalitaliano.org.ar

https://orcid.org/0000-0002-0612-320X Cómo citar este artículo: Gallucci G, Sánchez Saba J, Boretto JG, Zaidenberg EE, Donndorff A, Rellán I, De Carli P. Artroplastia total de codo después de un proceso infeccioso. Cirugía en dos tiempos. Rev Asoc Argent Ortop Traumatol 2021;86(2):185-196. https://doi.org/10.15417/issn. 1852-7434.2021.86.2.1160 


\section{INTRODUCCIÓN}

Se ha demostrado la utilidad de la artroplastia total de codo (ATC) para tratar enfermedades degenerativas y traumáticas del codo. Sin embargo, algunos cuadros, como las infecciones previas o la mala cobertura de los tejidos blandos, se han considerado una contraindicación estricta para el implante ${ }^{1-4}$ La colocación de una prótesis en el contexto de una infección previa representa un desafío, porque el proceso séptico inicial puede ser difícil de erradicar. La mayoría de las publicaciones describe el desarrollo de un proceso infeccioso después de una falla de la prótesis y, en estos casos, la bibliografía actual coincide en que la cirugía en dos etapas es el tratamiento de elección. ${ }^{4-7}$ Sin embargo, según nuestro conocimiento, hay muy pocos reportes sobre el tratamiento de las infecciones óseas de codo fuera del contexto de una prótesis. ${ }^{3,8}$

El objetivo de este artículo es comunicar los resultados de una cohorte de pacientes con un proceso infeccioso del codo que fueron tratados en dos etapas quirúrgicas: la primera con un espaciador de cemento con antibiótico (ECA) y la segunda con una ATC. Nuestro objetivo secundario fue determinar si la magnitud del defecto residual o la cantidad de procedimientos previos afectan el resultado final.

\section{MATERIALES Y MÉTODOS}

Se diseñó un estudio retrospectivo observacional de todos los pacientes sometidos a una ATC después de un proceso infeccioso, en nuestra institución, entre 2007 y 2019. Los criterios de inclusión fueron: pacientes $>18$ años, con un proceso infeccioso del codo, tratados en dos etapas quirúrgicas, la primera con ECA y la segunda con ATC; que ambos procedimientos hayan sido realizados en nuestra institución y por el mismo equipo quirúrgico, y un seguimiento $>1$ año.

La sospecha de infección se estableció mediante parámetros clínicos (eritema, edema, dolor, aumento de la temperatura local, fístula, exposición en caso de fracturas abiertas) y bioquímicos (eritrosedimentación, proteína $\mathrm{C}$ reactiva, recuento de glóbulos blancos). La confirmación infectológica se realizó mediante la toma de muestras para cultivos y anatomía patológica durante la cirugía.

Se registraron la causa de la cirugía primaria, el número de cirugías previas, el tiempo desde la cirugía primaria hasta la primera etapa quirúrgica, los gérmenes aislados, el tiempo de permanencia del ECA, el tiempo de antibioticoterapia, el defecto residual articular en el momento de la reconstrucción, el tipo de reconstrucción y si el paciente ya no tenía infección al final del seguimiento.

Para la evaluación objetiva se midió el rango de movilidad con un goniómetro y la evaluación subjetiva se realizó con los puntajes DASH ${ }^{9}$ (Disabilities of the Arm, Shoulder and Hand) y MEPS ${ }^{10}$ (Mayo Elbow Performance Score), la escala analógica visual (EAV) para dolor y satisfacción del paciente; y la fuerza de extensión del codo según la escala del MRC (Medical Research Council). ${ }^{11}$

Se tomaron radiografías de frente y de perfil, en el período posoperatorio inmediato, al mes, a los 3, 6 y 12 meses cada año, y al final del seguimiento. Se evaluó la presencia de aflojamientos según la escala de Morrey, ${ }^{12}$ que los clasifica en: grado 0 , línea radiolúcida $<1 \mathrm{~mm}$ y que envuelve $<50 \%$ de la interfaz; grado 1, línea radiolúcida de $1 \mathrm{~mm}$ y que envuelve $<50 \%$ de la interfaz; grado 2, línea radiolúcida $>1 \mathrm{~mm}$ y que envuelve $>50 \%$ de la interfaz; grado 3, línea radiolúcida $>2 \mathrm{~mm}$ y que envuelve toda la interfaz; grado 4, aflojamiento grosero. Se evaluó la presencia de calcificaciones heterotópicas mediante la clasificación de Hastings: ${ }^{13}$ clase I: osificación heterotópica en codo o antebrazo evidenciable radiográficamente, sin limitación funcional; clase IIA: limitación en el plano de flexión/extensión; clase IIB, limitación en el plano de pronación/supinación; clase IIC: limitación en ambos planos de movimiento; clase III: anquilosis que elimina la flexión/extensión del codo, la pronación/ supinación o ambas.

Como objetivo secundario, se evaluaron los resultados funcionales según el defecto óseo residual, y se agrupó a los pacientes en aquellos con un defecto $<4 \mathrm{~cm}$ (grupo A) o $>4 \mathrm{~cm}$ (grupo B) tanto en el cúbito como en el húmero. Esta división se hizo teniendo en cuenta que los acortamientos del húmero de $4 \mathrm{~cm}$ suelen ser compatibles con una buena función. ${ }^{14}$ La medición radiográfica se realizó antes de la segunda etapa quirúrgica.

\section{Técnica quirúrgica}

Primera etapa quirúrgica

Todos los pacientes fueron operados en decúbito dorsal con anestesia regional. Se empleó el abordaje posterior en ocho casos y el abordaje lateral, en dos. Siempre que se abordó por vía posterior se identificó y reparó el nervio 
cubital. Se llevó a cabo un meticuloso desbridamiento de los tejidos desvitalizados que incluyó la extracción de implantes y el cemento residual, si correspondía. Los canales medulares fueron liberados de toda seudomembrana e irrigados profusamente. Si no se contaba con un cultivo previo, se tomó una muestra durante la cirugía, para la estadificación, según los criterios de Mirra que evalúan el número de leucocitos polimorfonucleares por campo de gran aumento $(500 \mathrm{x}) \cdot{ }^{15}$ La muestra sugiere infección cuando se detectan 5 o más leucocitos polimorfonucleares por campo. Si no hay leucocitos polimorfonucleares, no existe ningún signo de infección tanto clínica como microbiológica.

Se enviaron varias muestras para cultivo diferido. Se confeccionó un espaciador de cemento al que se le agregaron $2 \mathrm{~g}$ de vancomicina por dosis de cemento en todos los casos y, en dos pacientes, además, se agregó teicoplanina $1 \mathrm{~g}$. En siete casos, el ECA se colocó con un clavo de Steinmann endomedular al cúbito y al húmero y, en tres casos, se insertó libre en el espacio articular.

Se evaluó el estado del tríceps para diseñar una táctica de reconstrucción posterior. Los pacientes fueron inmovilizados con una valva de yeso posterior y se colocó un catéter para administrar el antibiótico parenteral. Cuando se completó el tratamiento antibiótico, se dejó transcurrir dos semanas y se repitieron los análisis bioquímicos para determinar la eritrosedimentación y la proteína $\mathrm{C}$ reactiva. Ante parámetros compatibles con ausencia de infección, se indicó la reconstrucción.

\section{Segunda etapa quirúrgica}

El segundo tiempo operatorio se realizó a través de un abordaje posterior en todos los casos. Se extrajo el ECA y se enviaron muestras a anatomía patológica intraoperatoria y diferida. Una vez confirmada la ausencia de infección (según los criterios de Mirra), se realizó la reconstrucción articular. Dos pacientes necesitaron de un colgajo pediculado de dorsal ancho para la cobertura del defecto posterior. En cuatro casos, se colocó una aloprótesis ( 2 en el cúbito y 2 en el húmero). La fijación del aloinjerto al receptor fue a través de una placa LCP (Locking Compression Plate, Synthes TM, Oberdorf, Suiza) de 3,5 y 4,5 mm. El tríceps fue tratado de distintas maneras. En cinco pacientes, se desinsertó y se lo reinsertó posteriormente; en dos, se lo reparó; en un paciente, se suturó el tendón del tríceps al aloinjerto del cúbito; en otro, se suturó el tendón del dorsal pediculado al tríceps y, en otro, se suturó el tendón del dorsal pediculado al tendón del tríceps del aloinjerto cubital.

La prótesis se colocó de manera convencional. Siempre se utilizó una prótesis semiconstreñida. A seis pacientes se les colocó una prótesis de Coonrad/Morrey (Zimmer, Warsaw, IN, EE.UU.); a dos, una prótesis Discovery (Biomet, Warsaw, IN, EE.UU.) y, a dos, una prótesis Discovery-SRS (Biomet Orthopedics, Warsaw, IN, EE.UU.). Los pacientes fueron inmovilizados con una valva de yeso en extensión por tres semanas para permitir la cicatrización del tríceps.

\section{RESULTADOS}

Once pacientes fueron tratados durante este período. Uno fue excluido por pérdida en el seguimiento a los dos meses de la etapa de reconstrucción y, en ese momento, no tenía infección. El grupo final quedó constituido por 10 pacientes (4 hombres y 6 mujeres, edad promedio 62 años [rango 35-80]).

En la Tabla 1, se detallan las causas iniciales, la cantidad de cirugías y los tratamientos previos, el tipo de gérmenes, los defectos óseos y los tipos de reconstrucción.

En ocho de los 10 casos, fue posible obtener muestras de laboratorio para reactantes de fase aguda (eritrosedimentación y proteína $\mathrm{C}$ reactiva) antes de la artroplastia y los valores fueron patológicos.

A todos los pacientes se les realizó una punción biopsia previa, que fue positiva en nueve de ellos. No fue posible aislar el germen en un paciente y se llegó al diagnóstico por la presencia de más de 5 leucocitos polimorfonucleares por campo en la biopsia intraoperatoria y la anatomía patológica diferida (caso 1).

En la segunda etapa quirúrgica, se tomaron biopsias intraoperatorias siguiendo los criterios de Mirra que indicaron $<5$ leucocitos polimorfonucleares por campo en todos los casos.

El seguimiento promedio desde la primera etapa quirúrgica fue de 5 años (rango 1-10).

En cinco pacientes, no se pudo evaluar el rango de movilidad previo: en tres por tratarse de resecciones articulares amplias y completamente inestables y, en dos, por ser fracturas con gran pérdida del stock óseo. Los resultados se muestran en la Tabla 2. 
Tabla 1. Datos demográficos

\begin{tabular}{|c|c|c|c|c|c|c|c|c|c|c|}
\hline Paciente & Edad & Sexo & Causa & $\begin{array}{c}\text { Tratamiento } \\
\text { previo }\end{array}$ & $\begin{array}{l}\text { Cirugías } \\
\text { previas }\end{array}$ & $\begin{array}{c}\text { Tiempo } \\
\text { hasta la } \\
\text { primera } \\
\text { etapa }\end{array}$ & Cultivo & $\begin{array}{c}\text { Tiempo } \\
1-2 \\
\text { etapa }\end{array}$ & $\begin{array}{c}\text { Grupo } \\
\text { (defecto, } \\
\text { cm) }\end{array}$ & $\begin{array}{l}\text { Tipo de } \\
\text { reconstruc- } \\
\text { ción }\end{array}$ \\
\hline 1 & 80 & M & AP & Infiltración & 1 & 11 meses & Negativo & 3 & $\begin{array}{c}\text { A } \\
(3 \mathrm{~cm})\end{array}$ & $\begin{array}{c}\text { ATC } \\
\text { (Coonrad/ } \\
\text { Morrey) }\end{array}$ \\
\hline 2 & 65 & $\mathrm{~F}$ & $\mathrm{AR}$ & Sinovectomía & 2 & 14 meses & $\begin{array}{l}\text { S. aureus } \\
\text { sensible a } \\
\text { meticilina }\end{array}$ & 3 & $\begin{array}{c}\text { A } \\
(2 \mathrm{~cm})\end{array}$ & $\begin{array}{c}\text { ATC } \\
\text { (Coonrad/ } \\
\text { Morrey) }\end{array}$ \\
\hline 3 & 69 & $\mathrm{~F}$ & $\begin{array}{l}\text { Fractura } \\
\text { supracon- } \\
\text { dílea }\end{array}$ & $\begin{array}{l}\text { Osteosíntesis/ } \\
\text { limpieza/ATC }\end{array}$ & 8 & 5 años & $\begin{array}{l}\text { Polimicro- } \\
\text { biano }\end{array}$ & 2 & $\begin{array}{c}\text { B } \\
(16 \mathrm{~cm})\end{array}$ & $\begin{array}{l}\text { Aloprótesis } \\
\text { de cúbito } \\
\text { (Coonrad/ } \\
\text { Morrey) }\end{array}$ \\
\hline 4 & 61 & $\mathrm{~F}$ & Luxación & $\begin{array}{c}\text { Plástica } \\
\text { ligamentaria }\end{array}$ & 2 & 9 meses & $\begin{array}{l}\text { Estafi- } \\
\text { lococo } \\
\text { coagulasa } \\
\text { negativo }\end{array}$ & 2,5 & $\begin{array}{c}\text { A } \\
(2 \mathrm{~cm})\end{array}$ & $\begin{array}{c}\text { ATC } \\
\text { (Coonrad/ } \\
\text { Morrey) }\end{array}$ \\
\hline 5 & 60 & M & $\begin{array}{l}\text { Fractura } \\
\text { supracon- } \\
\text { dílea }\end{array}$ & ATC/artrólisis & 2 & 1 año & $\begin{array}{l}\text { S. epider- } \\
\quad \text { midis }\end{array}$ & 3 & $\begin{array}{c}\text { A } \\
(3 \mathrm{~cm})\end{array}$ & $\begin{array}{l}\text { ATC (Coon- } \\
\mathrm{rad} / \text { Morrey) }\end{array}$ \\
\hline 6 & 35 & M & $\begin{array}{l}\text { Fractura } \\
\text { supracon- } \\
\text { dílea }\end{array}$ & Limpieza & 1 & 2 meses & $\begin{array}{l}S . \text { epider- } \\
\text { midis }\end{array}$ & 3 & $\begin{array}{c}\text { B } \\
(7 \mathrm{~cm})\end{array}$ & $\begin{array}{l}\text { Aloprótesis } \\
\text { de húmero } \\
\text { (Coonrad/ } \\
\text { Morrey) }\end{array}$ \\
\hline 7 & 71 & $\mathrm{~F}$ & $\begin{array}{l}\text { Fractura } \\
\text { supracon- } \\
\text { dílea }\end{array}$ & $\begin{array}{l}\text { Osteosíntesis/ } \\
\text { limpieza/ATC }\end{array}$ & 4 & 4 años & $\begin{array}{l}S . \text { epider- } \\
\quad \text { midis }\end{array}$ & 8 & $\begin{array}{c}\text { B } \\
(10 \mathrm{~cm})\end{array}$ & $\begin{array}{c}\text { ATC } \\
\text { (Discovery- } \\
\text { SRS) }\end{array}$ \\
\hline 8 & 60 & $\mathrm{~F}$ & $\begin{array}{l}\text { Fractura } \\
\text { supracon- } \\
\text { dílea }\end{array}$ & $\begin{array}{l}\text { Osteosíntesis/ } \\
\text { limpieza/ATC }\end{array}$ & 19 & 29 años & $\begin{array}{l}\text { Polimicro- } \\
\text { biano }\end{array}$ & 3 & $\begin{array}{c}\text { B } \\
(23 \mathrm{~cm})\end{array}$ & $\begin{array}{l}\text { Aloprótesis } \\
\text { de cúbito } \\
\text { (Discovery- } \\
\text { SRS) + dorsal } \\
\text { ancho }\end{array}$ \\
\hline 9 & 51 & $\mathrm{~F}$ & $\begin{array}{l}\text { Fractura } \\
\text { supracon- } \\
\text { dílea }\end{array}$ & Limpieza & 2 & $1 \mathrm{mes}$ & $\begin{array}{l}\text { Polimicro- } \\
\text { biano }\end{array}$ & 4 & $\begin{array}{c}\text { B } \\
(15 \mathrm{~cm})\end{array}$ & $\begin{array}{l}\text { Aloprótesis } \\
\text { de húmero } \\
\text { (Discovery) + } \\
\text { dorsal ancho }\end{array}$ \\
\hline 10 & 63 & M & $\begin{array}{l}\text { Tríada } \\
\text { terrible }\end{array}$ & $\begin{array}{l}\text { Plástica liga- } \\
\text { mentaria }\end{array}$ & 2 & 4 meses & $\begin{array}{l}\text { S. aureus } \\
\text { resistente } \\
\text { a metici- } \\
\quad \text { lina }\end{array}$ & 5 & $\begin{array}{c}\text { A } \\
(2 \mathrm{~cm})\end{array}$ & ATC (C-M) \\
\hline Promedio & 61 & & & & 4,3 & & & 3,6 & 8,3 & \\
\hline
\end{tabular}

$\mathrm{M}=$ masculino, $\mathrm{F}=$ femenino, $\mathrm{ATC}=$ artroplastia total de codo, $\mathrm{AP}=$ artritis psoriásica, $\mathrm{AR}=$ artritis reumatoide. 
Tabla 2. Resultados

\begin{tabular}{|c|c|c|c|c|c|c|c|c|c|c|c|c|c|c|}
\hline \multirow[t]{2}{*}{ Paciente } & \multirow{2}{*}{$\begin{array}{l}\text { Segui- } \\
\text { miento } \\
\text { (años) }\end{array}$} & \multicolumn{2}{|c|}{ Flexo-extensión } & \multicolumn{2}{|c|}{ Dolor } & \multicolumn{2}{|c|}{ MEPS } & \multicolumn{2}{|c|}{ DASH } & \multirow{2}{*}{$\begin{array}{c}\text { Fuer- } \\
\text { za de } \\
\text { exten- } \\
\text { sión }\end{array}$} & \multirow{2}{*}{$\begin{array}{l}\text { Reso- } \\
\text { lución } \\
\text { de la } \\
\text { infec- } \\
\text { ción }\end{array}$} & \multirow{2}{*}{$\begin{array}{l}\text { Afloja- } \\
\text { miento } \\
\text { de la } \\
\text { prótesis }\end{array}$} & \multirow{2}{*}{$\begin{array}{l}\text { Cal- } \\
\text { cifica- } \\
\text { ciones } \\
\text { hetero- } \\
\text { tópicas }\end{array}$} & \multirow{2}{*}{$\begin{array}{l}\text { Compli- } \\
\text { caciones }\end{array}$} \\
\hline & & $\begin{array}{c}\text { Pre- } \\
\text { operato- } \\
\text { ria }\end{array}$ & $\begin{array}{l}\text { Pos- } \\
\text { operato- } \\
\text { ria }\end{array}$ & $\begin{array}{c}\text { Pre- } \\
\text { opera- } \\
\text { torio }\end{array}$ & $\begin{array}{l}\text { Pos- } \\
\text { opera- } \\
\text { torio }\end{array}$ & $\begin{array}{c}\text { Preo- } \\
\text { perato- } \\
\text { rio }\end{array}$ & $\begin{array}{l}\text { Pos- } \\
\text { opera- } \\
\text { torio }\end{array}$ & $\begin{array}{l}\text { Pre- } \\
\text { opera- } \\
\text { torio }\end{array}$ & $\begin{array}{c}\text { Pos- } \\
\text { opera- } \\
\text { torio }\end{array}$ & & & & & \\
\hline 1 & 10 & $120 / 30$ & $135 / 10$ & 7 & 0 & 45 & 100 & 28 & 20 & M5 & Sí & No & No & No \\
\hline 2 & 9.5 & $130 / 30$ & $140 / 35$ & 6 & 0 & 60 & 75 & 72 & 33 & M4 & Sí & 1 & No & No \\
\hline 3 & 5.3 & $\begin{array}{c}\text { No } \\
\text { evaluada }\end{array}$ & $125 / 30$ & 8 & 3 & 20 & 75 & 68 & 26 & M3 & Sí & No & No & No \\
\hline 4 & 5 & $110 / 30$ & $140 / 25$ & 7 & 4 & 40 & 90 & 39 & 50 & M5 & Sí & No & No & $\begin{array}{c}\text { Afloja- } \\
\text { miento } \\
\text { de pernos }\end{array}$ \\
\hline 5 & 4.5 & $110 / 30$ & $130 / 15$ & 5 & 1 & 45 & 100 & 46 & 20 & M5 & Sí & No & No & No \\
\hline 6 & 9 & $\begin{array}{c}\text { No } \\
\text { evaluada }\end{array}$ & $135 / 40$ & --- & 1 & --- & 70 & & 24 & M4 & Sí & 2 & No & No \\
\hline 7 & 1.7 & $\begin{array}{c}\text { No } \\
\text { evaluada }\end{array}$ & $110 / 40$ & 5 & 2 & 50 & 75 & 65 & 23 & M0 & Sí & 2 & No & No \\
\hline 8 & 1 & $\begin{array}{c}\text { No } \\
\text { evaluada }\end{array}$ & $120 / 30$ & 6 & 7 & 20 & 40 & 72 & 58 & M1 & No & 3 & No & $\begin{array}{c}\text { Necrosis } \\
\text { del } \\
\text { colgajo }\end{array}$ \\
\hline 9 & 2.3 & $\begin{array}{c}\text { No } \\
\text { evaluada }\end{array}$ & $140 / 30$ & --- & 2 & --- & 85 & - & 23 & M1 & Sí & No & I & No \\
\hline 10 & 1 & $115 / 25$ & $120 / 30$ & 8 & 5 & 35 & 80 & 58 & 27 & M5 & Sí & 1 & IIC & No \\
\hline $\begin{array}{c}\text { Pro- } \\
\text { medio }\end{array}$ & 5 & $117 / 29$ & $130 / 29$ & 6,5 & 2,5 & 40 & 80 & 56 & 30 & & & & & \\
\hline
\end{tabular}

DASH (Disabilities of the Arm, Shoulder and Hand), MEPS (Mayo Elbow Performance Score).

Un paciente (caso 4) presentó un aflojamiento de los pernos por una falla técnica en la inserción a los dos meses de la cirugía, y necesitó una nueva operación para su correcta colocación (Figura 1). Por otro lado, se detectó una necrosis del colgajo con recurrencia de la infección en un paciente (caso 8). Salvo este último paciente, todos tuvieron parámetros bioquímicos normales en el posoperatorio y no se detectó una reactivación de la infección en el último seguimiento.

$\mathrm{Al}$ evaluar a los pacientes según su stock óseo, cinco fueron incluidos dentro del grupo A y cinco, dentro del grupo B (Figuras 2 y 3). El grupo A tenía menos cirugías previas y obtuvo mejores resultados funcionales, principalmente en la recuperación de la fuerza de extensión, que el grupo B (Tabla 3). 

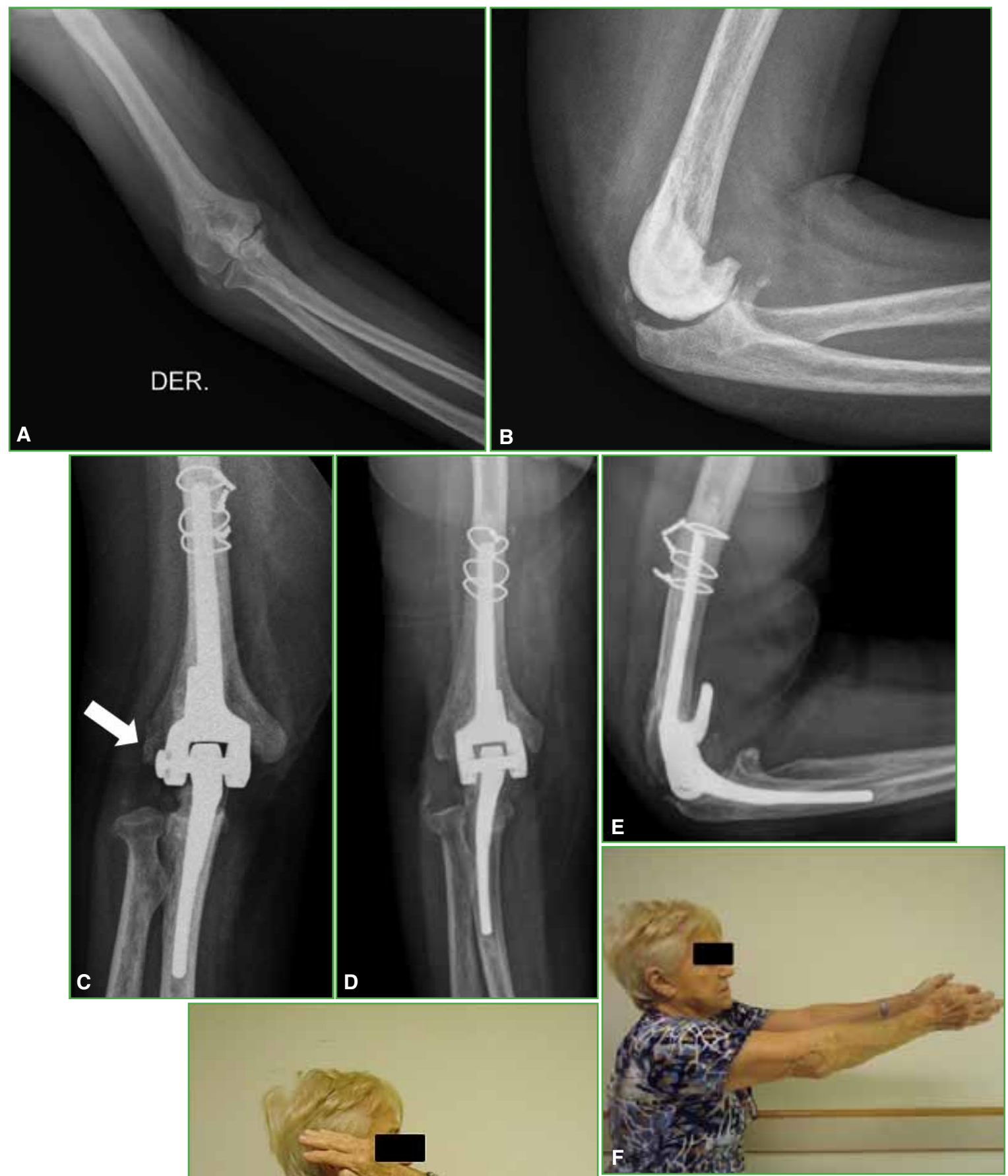

Figura 1. A. Radiografía de una mujer de 63 años (caso 4) a los 9 meses de la cirugía por inestabilidad del codo con desgate articular e infección. B. Espaciador de cemento con antibiótico. C. Radiografía a los 2 meses con aflojamiento de los pernos de la prótesis. D y E. Radiografías a los 3 años de la cirugía. F y G. Movilidad final. 

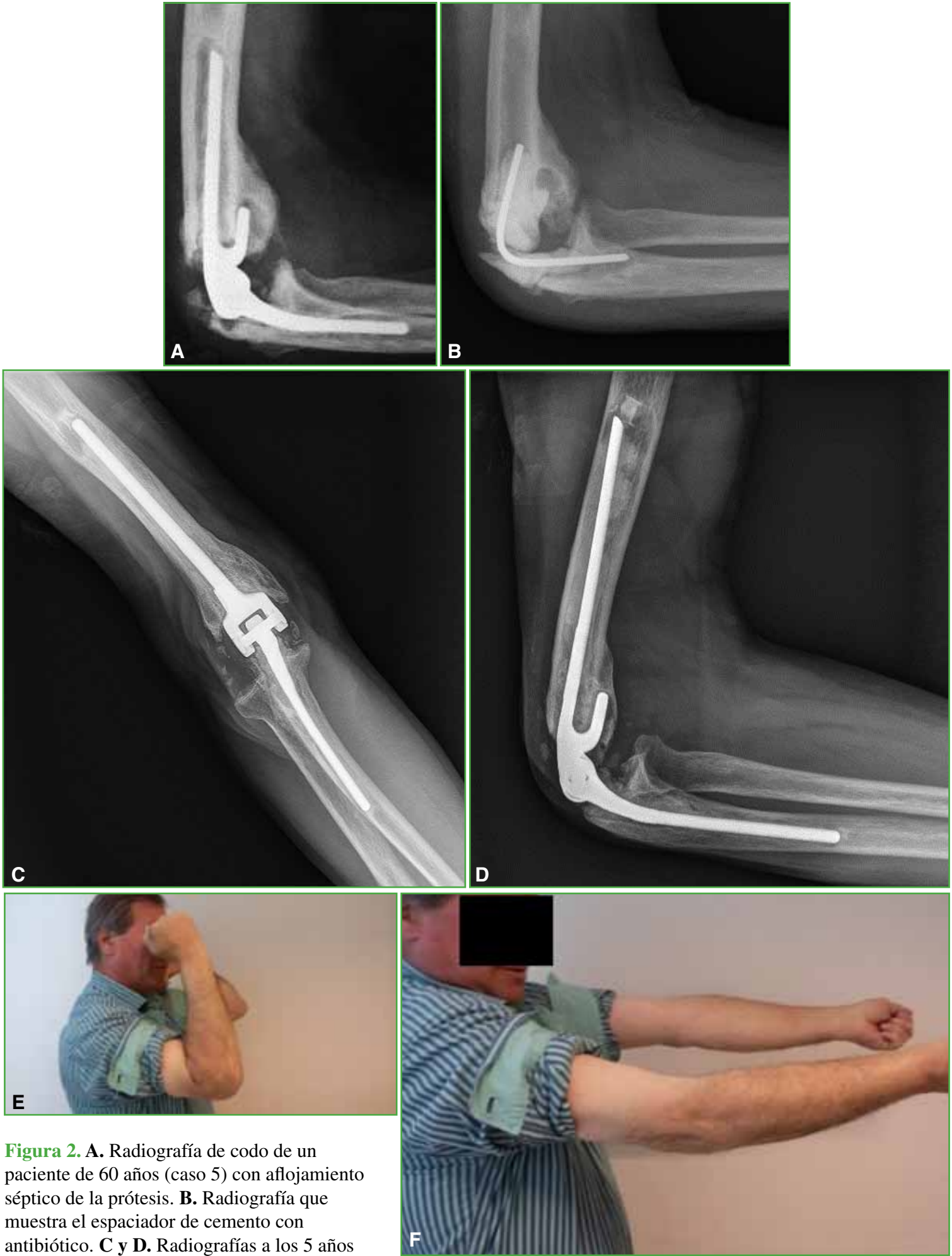
de la cirugía. E y F. Movilidad final. 

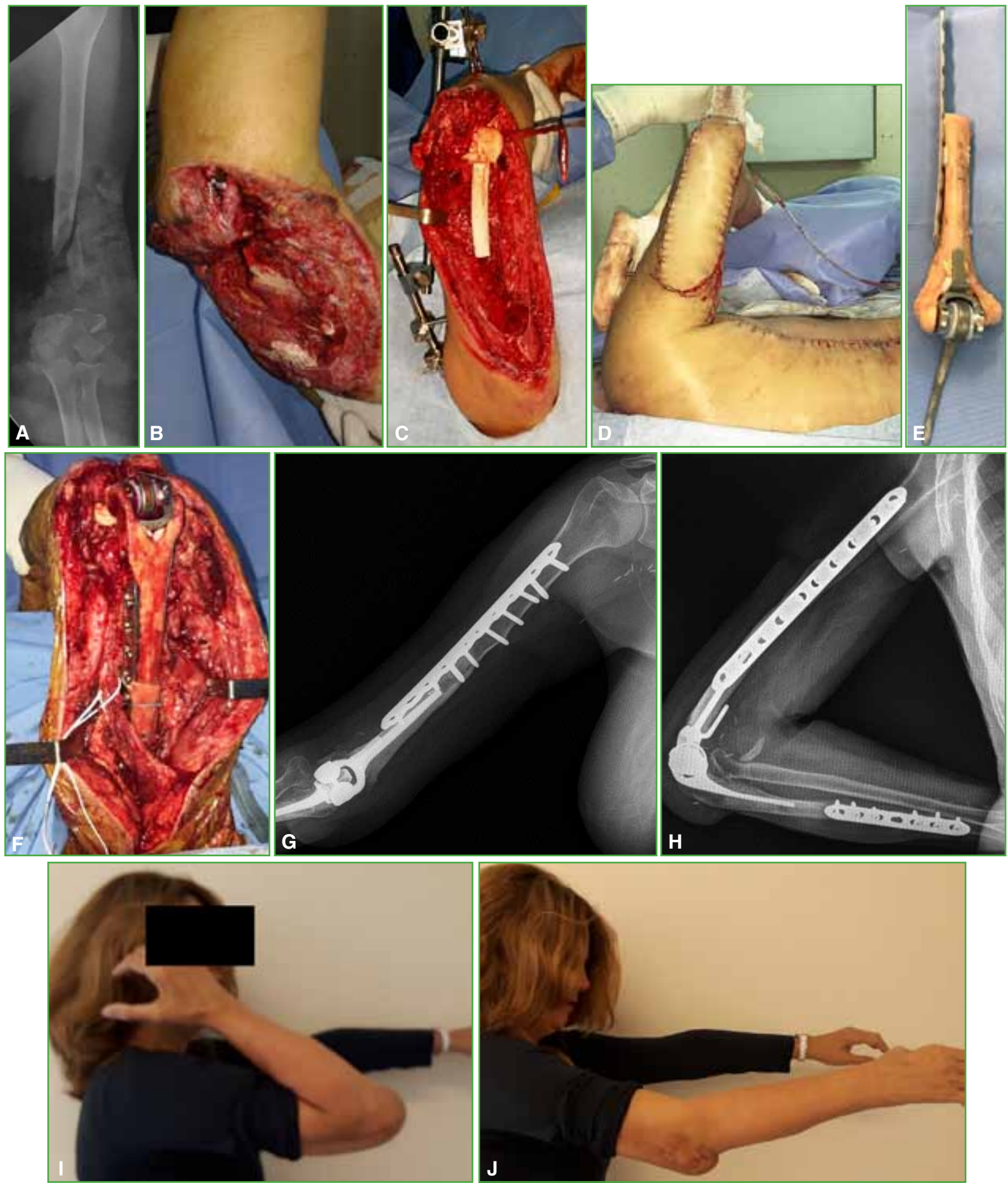

Figura 3. A. Radiografía de húmero de una mujer de 51 años (caso 9) con secuela de fractura expuesta infectada de un mes de evolución. Se observa la gran pérdida de stock óseo. B. Pérdida de partes blandas posterior (incluido el aparato extensor). C. Espaciador de cemento con antibiótico y tutor externo. D. Colgajo de dorsal ancho.

E. Preparación de la aloprótesis de húmero distal. F. Aloprótesis colocada con buena compresión en la osteotomía. G y H. Radiografía a los 2 años de la cirugía. I y J. Movilidad final. 
Tabla 3. Comparación entre los grupos A y B

\begin{tabular}{|c|c|c|c|c|}
\hline & \multicolumn{2}{|c|}{ Grupo A } & \multicolumn{2}{|c|}{ Grupo B } \\
\hline Cantidad de pacientes & \multicolumn{2}{|c|}{5} & \multicolumn{2}{|c|}{5} \\
\hline Edad & \multicolumn{2}{|c|}{67 (rango 60-80) } & \multicolumn{2}{|c|}{56 (rango 35-69) } \\
\hline Cirugías previas & \multicolumn{2}{|c|}{2,2 (rango 1-4) } & \multicolumn{2}{|c|}{ 6,4 (rango 1-19) } \\
\hline \multirow[t]{2}{*}{ Dolor } & Preoperatorio & 6,4 (rango 5-8) & Preoperatorio & 6,6 (rango 6-8) \\
\hline & Posoperatorio & 2,4 (rango 0-5) & Posoperatorio & 2,6 (rango 0-7) \\
\hline \multirow[t]{2}{*}{ DASH } & Preoperatorio & 47 (rango 28-65) & Preoperatorio & 57 (rango 33-72) \\
\hline & Posoperatorio & 28 (rango 20-50) & Posoperatorio & 41 (rango 23-72) \\
\hline \multirow[t]{2}{*}{ MEPS } & Preoperatorio & 43 (rango 35-60) & Preoperatorio & 33 (rango 20-60) \\
\hline & Posoperatorio & 92 (rango 80-100) & Posoperatorio & $69($ rango $40-85)$ \\
\hline Fuerza de tríceps & Posoperatorio & $\begin{array}{l}4 \times \mathrm{M} 5 \\
1 \times \mathrm{M} 4\end{array}$ & Posoperatorio & $\begin{array}{l}1 \times M 41 \times M 3 \\
2 \times M 11 \times M 0\end{array}$ \\
\hline Satisfacción & \multicolumn{2}{|c|}{8 (rango 5-10) } & \multicolumn{2}{|c|}{6 (rango 2-8) } \\
\hline
\end{tabular}

DASH (Disabilities of the Arm, Shoulder and Hand), MEPS (Mayo Elbow Performance Score).

\section{DISCUSIÓN}

El codo es una articulación con una limitada cobertura de tejidos blandos y, por lo tanto, más propensa a procesos infecciosos en comparación con otras articulaciones. ${ }^{16-19}$ Desde hace años, se considera a la osteomielitis como una contraindicación para la colocación de una prótesis. Sin embargo, el gran avance en el tratamiento de las infecciones, principalmente en la revisión de prótesis de cadera y rodilla, determinó que esa contraindicación sea, hoy en día, menos frecuente.

Cuando se analiza la bibliografía sobre las causas de los procesos infecciosos en el codo, la artroplastia surge como la causa desencadenante más común. Tan es así que existen pocos reportes de una ATC después de un episodio degenerativo o traumático no protésico.

Nuestra casuística incluye seis de los pacientes con osteomielitis que no habían sido tratados con prótesis y, en los cuatro restantes, la ATC había sido parte de alguno de sus tratamientos previos.

La cirugía en dos tiempos es el tratamiento de elección para pacientes con procesos infecciosos articulares. Peach y cols. ${ }^{20}$ comunicaron buenos resultados con el tratamiento en dos tiempos de 33 pacientes con prótesis infectadas y una tasa baja de reinfección. En una revisión sistemática de 309 ATC, Gutman y cols. ${ }^{21}$ identificaron una tasa de curación de la infección del 81,2\% con una cirugía en dos tiempos. Zmistowski y cols. ${ }^{19}$ reportaron 26 pacientes con infecciones de la prótesis. Diez de ellos fueron sometidos a un desbridamiento con retención de los implantes y 16, a una cirugía en dos tiempos con ECA. Cinco de los primeros 10 pacientes desarrollaron una infección, mientras que, en el segundo grupo, tres de los 16 pacientes evolucionaron desfavorablemente.

La artroplastia por resección sin ECA es una opción terapéutica en cuadros de infección. ${ }^{22-24}$ Yamaguchi y cols. ${ }^{3}$ publicaron los resultados de 10 pacientes sometidos a una ATC después de un proceso infeccioso que inicialmente había sido tratado con una artroplastia por resección. Siete pacientes tenían una prótesis previa y tres casos correspondían a artritis séptica y una fractura de húmero distal infectada. El tiempo promedio desde la artroplastia de resección hasta el implante fue de 3.8 años. Ocho de los pacientes no tenían infección al final del seguimiento y dos sufrieron la reactivación del proceso infeccioso.

La implantación de un ECA después del desbridamiento en un paciente con osteomielitis no solo ayuda a obtener una menor concentración de gérmenes en el sitio, sino que además permite la formación de una seudomembrana sinovial alrededor de este. ${ }^{25}$ Los beneficios de esta seudomembrana fueron informados por Pelissier y cols. ${ }^{26}$ quienes demostraron la producción, a partir de esta, de factores de crecimiento y factores osteoinductores capaces de diferenciarse en células de la línea osteoblástica. Además, el espaciador provee cierta estabilidad que, junto con el antibiótico específico, es fundamental para curar la infección. 
La infección en la articulación del codo afecta, con frecuencia, las partes blandas y los resultados funcionales de una prótesis no son comparables con aquellas prótesis que no han sufrido una infección.

Kwak y cols. ${ }^{6}$ evaluaron los resultados radiológicos y clínicos en pacientes que habían sido sometidos a una revisión de la ATC por causas infecciosas y no infecciosas. En este estudio, hallaron que el resultado clínico fue inferior en el grupo infectado, y que los pacientes con períodos de espera más prolongados hasta la colocación del implante obtenían peores resultados clínicos.

Nuestra investigación arrojó resultados similares. Los pacientes con una evolución larga y artroplastias por resección debido a una infección recurrente y defectos óseos más grandes y múltiples cirugías obtenían peores resultados funcionales, pese a la remisión del proceso infeccioso.

La erradicación de todo el tejido infectado es primordial en el tratamiento de una infección. Esto puede llevar a la necesidad de una resección amplia de hueso y tejidos blandos. Los implantes previos o las prótesis flojas, a menudo, producen osteólisis marcada que aumenta la pérdida de stock óseo y, en general, cuanto mayor es el defecto óseo residual, mayor es la lesión de partes blandas que lo acompaña. El tríceps es particularmente susceptible en este tipo de escenarios y, por eso, es tan frecuente su insuficiencia a pesar de los intentos de reconstrucción. Dukin y cols. ${ }^{27}$ analizaron a 93 pacientes después de una ATC infectada y observaron una debilidad final del tríceps en el 55\% de los casos. Esto demuestra la importancia de conservar el aparato extensor en este tipo de situacionesNuestro estudio tiene varias limitaciones, como el diseño retrospectivo, la limitada cantidad de pacientes, la heterogeneidad de las causas iniciales, la variedad de los defectos residuales, el corte en $4 \mathrm{~cm}$ de defecto óseo para la separación de los grupos (sin una verdadera base científica) y el corto seguimiento para un reemplazo de prótesis. Sin embargo y, a diferencia de otros estudios, nuestra casuística incluye muchos pacientes con causas no protésicas previas, lo que no está ampliamente reportado en la literatura.

El grado de curación de la infección fue alto ( 9 de 10 pacientes), pero sin una función satisfactoria en todos los casos. Cuanto mayor fue la resección ósea y, por ende, mayor la lesión de partes blandas, peores fueron los resultados funcionales, sobre todo en cuanto a la recuperación de la fuerza de extensión.

\section{CONCLUSIONES}

El tratamiento de un proceso infeccioso del codo mediante un meticuloso desbridamiento asociado a la colocación de un ECA permite controlar la infección en un alto porcentaje de los casos. La reconstrucción secundaria es demandante y se asocia con complicaciones principalmente en la dificultosa recuperación de la extensión. Es de esperar que, cuanto mayor sea el defecto óseo y mayor la cantidad de procedimientos previos, los resultados funcionales sean peores.

Conflicto de intereses: Los autores no declaran conflictos de intereses.

ORCID de J. Sánchez Saba: https://orcid.org/0000-0001-5496-3513

ORCID de J. G. Boretto: https://orcid.org/0000-0001-7701-3852

ORCID de E. E. Zaidenberg: https://orcid.org/0000-0002-1535-0586
ORCID de A. Donndorff: https://orcid.org/0000-0002-6384-4820

ORCID de I. Rellán: https://orcid.org/0000-0003-4045-339X

ORCID de P. De Carli: https://orcid.org/0000-0002-0612-320X

\section{BIBLIOGRAFÍA}

1. Kraay MJ, Figgie MP, Inglis AE, Wolfe SW, Ranawat CS. Primary semiconstrained total elbow arthroplasty. Survival analysis of 113 consecutive cases. J Bone Joint Surg Br 1994;76(4):636-40. PMID: 8027155

2. Wolfe SW, Figgie MP, Inglis AE, Bohn WW, Ranawat CS. Management of infection about total elbow prostheses. $J$ Bone Joint Surg Am 1990;72(2):198-212. PMID: 2303506

3. Yamaguchi K, Adams RA, Morrey BF. Semiconstrained total elbow arthroplasty in the context of treated previous infection. J Shoulder Elbow Surg 1999;8(5):461-5. https://doi.org/10.1016/s1058-2746(99)90077-0 
4. Rudge WBJ, Eseonu K, Brown M, Warren S, Majed A, Bayley IL, et al. The management of infected elbow arthroplasty by two-stage revision. J Shoulder Elbow Surg 2018;27(5):879-86. https://doi.org/10.1016/j.jse.2017.12.033

5. Kunutsor SK, Beswick AD, Whitehouse MR, Blom AW. One- and two-stage surgical revision of infected elbow prostheses following total joint replacement: a systematic review. BMC Musculoskelet Disord 2019;20(1):467. https://doi.org/10.1186/s12891-019-2848-x

6. Kwak JM, Kholinne E, Sun Y, Kim MS, Koh KH, Jeon IH. Clinical results of revision total elbow arthroplasty: comparison of infected and non-infected total elbow arthroplasty. Int Orthop 2019;43(6):1421-7. https://doi.org/10.1007/s00264-018-4267-2

7. Watts AC, Duckworth AD, Trail IA, Rees J, Thomas M, Rangan A. Scoping review: Diagnosis and management of periprosthetic joint infection in elbow arthroplasty. Shoulder Elbow 2019;11(4):282-91. https://doi.org/10.1177/1758573218789341

8. Gallucci G, Gonzalez D, Boretto J, Alfie V, Donndorff A, De Carli P. Artroplastía total del codo posterior a una artritis séptica. Rev Asoc Argent Ortop Traumatol 2010;75(3):287-92. https://www.aaot.org.ar/revista/2010/n3/Rev_AsocArgentOrtopTraumatol_2010_75_(3)_287.pdf

9. Hudak PL, Amadio PC, Bombardier C. Development of an upper extremity outcome measure: the DASH (disabilities of the arm, shoulder and hand) [corrected]. The Upper Extremity Collaborative Group (UECG). Am J Ind Med 1996;29:602-8. https://doi.org/10.1002/(SICI)1097-0274(199606)29:6<602::AID-AJIM4>3.0.CO;2-L

10. Morrey B. Post-traumatic contracture of the elbow. Operative treatment, including distraction arthroplasty. $J$ Bone $J$ Surg Am 1990;72:601-18. PMID: 2324148

11. Florence JM, Pandya S, King WM, J D Robison, J Baty, J P Miller, et al. Intrarater reliability of manual muscle test (Medical Research Council scale) grades in Duchenne's muscular dystrophy. Phys Ther 1992;72(2):115-126. https://doi.org/10.1093/ptj/72.2.115

12. Morrey BF, Bryan RS, Dobyns JH, Linscheld RL. Total elbow arthroplasty. A five-year experience at the Mayo Clinic. J Bone Joint Surg Am 1981;63:1050-63. PMID: 7276042

13. Hastings $\mathrm{H}$ 2nd, Graham TJ. The classification and treatment of heterotopic ossification about the elbow and forearm. Hand Clin 1994;10(3):417-37. PMID: 7962148

14. Kusnezov N, Dunn JC, Stewart J, Mitchell JS, Pirela-Cruz M. Acute limb shortening for major near and complete upper extremity amputations with associated neurovascular injury: a review of the literature. Orthop Surg 2015;7(4):306-16. https://doi.org/10.1111/os.12213

15. Mirra JM, Maeder RA, Amstutz HC. The pathology of failed total joint arthroplasty. Clin Orthop 1982;170:175-83. PMID: 7127944

16. Moro JK, King GJ. Total elbow arthroplasty in the treatment of posttraumatic conditions of the elbow. Clin Orthop Relat Res 2000;(370):102-14. https://doi.org/10.1097/00003086-200001000-00010

17. Lovy AJ, Keswani A, Dowdell J, Koehler S, Kim J, Hausman MR. Outcomes, complications, utilization trends, and risk factors for primary and revision total elbow replacement. J Shoulder Elbow Surg 2016;25(6):1020-1026. https://doi.org/10.1016/j.jse.2015.12.012

18. Prkic A, Welsink C, The B, van den Bekerom MPJ, Eygendaal D. Why does total elbow arthroplasty fail today? A systematic review of recent literature. Arch Orthop Trauma Surg 2017;137(6):761-9. https://doi.org/10.1007/s00402-017-2687-x

19. Zmistowski B, Pourjafari A, Padegimas EM, Sheth M, Cox RM, Ramsey ML, et al. Treatment of periprosthetic joint infection of the elbow: 15-year experience at a single institution. J Shoulder Elbow Surg 2018;27(9):1636-41. https://doi.org/10.1016/j.jse.2018.05.035

20. Peach CA, Nicoletti S, Lawrence TM, Stanley D. Two-stage revision for the treatment of the infected total elbow arthroplasty. Bone Joint J Br 2013;95(12):1681-6. https://doi.org/10.1302/0301-620X.95B12.31336

21. Gutman MJ, Stone MA, Namdari S, Abboud JA. Treatment of elbow periprosthetic joint infection: a systematic review of clinical outcomes. J Shoulder Elbow Surg 2020;29(2):411-9. https://doi.org/10.1016/j.jse.2019.10.002

22. Rhee YG, Cho NS, Park JG, Song JH. Resection arthroplasty for periprosthetic infection after total elbow arthroplasty. J Shoulder Elbow Surg 2016;25(1):105-11. https://doi.org/10.1016/j.jse.2015.08.045

23. Cheung EV, Adams RA, Morrey BF. Reimplantation of a total elbow prosthesis following resection arthroplasty for infection. J Bone Joint Surg Am 2008;90(3):589-94. https://doi.org/10.2106/JBJS.F.00829 
24. Allende C, Mangupli M, Bagliardelli J, Díaz P, Allende BT. Infected nonunions of long bones of the upper extremity: staged reconstruction using polimethylmethacrylate and bone graft impregnated with antibiotics. Chir Organi Mov 2009;93(3):137-42. https://doi.org/10.1007/s12306-009-0046-y

25. Somerson JS, Morrey ME, Sanchez-Sotelo J, Morrey BF. Diagnosis and management of periprosthetic elbow infection. J Bone Joint Surg Am 2015;97(23):1962-71. https://doi.org/10.2106/JBJS.O.00170

26. Pelissier A, Masquelet R, Bareille S. Induced membranes secrete growth factors including vascular and osteoinductive factors and could stimulate bone regeneration. J Orthop Res 2004;22:73-9. https://doi.org/10.1016/S0736-0266(03)00165-7

27. Duquin TR, Jacobson JA, Schleck CD, Larson DR, Sanchez-Sotelo J, Morrey BF. Triceps insufficiency after the treatment of deep infection following total elbow replacement. Bone Joint J Br 2014;96(1):82-7. https://doi.org/10.1302/0301-620X.96B1.31127 